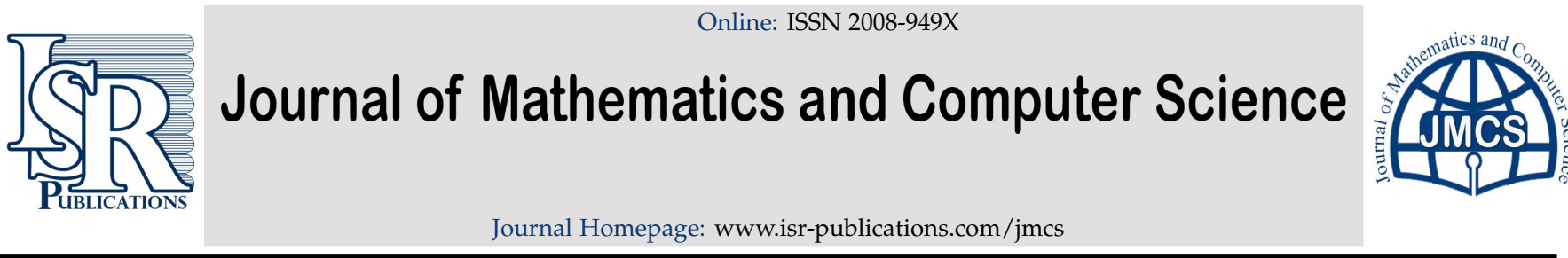

\title{
Oscillation criteria for a class of third order neutral dis- tributed delay differential equations with damping
}

\author{
M. H. Weia, ${ }^{a,}$ M. L. Zhang ${ }^{a}$, X. L. Liu ${ }^{a}$, Y. H. Yu ${ }^{b}$ \\ a School of Mathematics and Statistics, Yulin University, Yulin 719000, China. \\ ${ }^{b}$ Academy of Mathematics and System Sciences, Chinese Academy of Sciences, Beijing 100190, China.
}

\begin{abstract}
In this paper, the oscillation criteria of a class of third order neutral distributed delay differential equations with damping are investigated. This work is the continuation of the study by Saker [S. H. Saker, Math. Slovaca, 56 (2006), 433-450] and the extension of the work by Zhang [Q. X. Zhang, L. Gao, Y. H. Yu, Appl. Math. Lett., 25 (2012), 1514-1519] on oscillation properties of nonlinear third order delay differential equation. By choosing the appropriate functions and using a generalized Riccati transformation, some new oscillation criteria are presented to insure that every solution of this equation oscillates or converges to zero. The presented results correct and improve the earlier ones in existing literature. Finally, several illustrative examples are included.
\end{abstract}

Keywords: Oscillation criteria, third order, distributed delay, damping, Riccati transformation.

2010 MSC: 34C10, 34K11.

(C)2019 All rights reserved.

\section{Introduction}

In this article, we consider third order neutral distributed delay differential equations with damping of the form

$$
\begin{aligned}
& \left(r_{1}(t)\left(r_{2}(t)\left(x(t)+\int_{a}^{b} p(t, \mu) x(\sigma(t, \mu)) d \mu\right)^{\prime}\right)^{\prime}\right)^{\prime} \\
& \quad+m(t)\left(r_{2}(t)\left(x(t)+\int_{a}^{b} p(t, \mu) x(\sigma(t, \mu)) d \mu\right)^{\prime}\right)^{\prime}+\int_{c}^{d} q(t, \zeta) f(x(\tau(t, \zeta))) d \zeta=0 .
\end{aligned}
$$

Throughout the whole paper, we assume that the following hypotheses hold:

$\left(C_{1}\right) r_{1}(t) \in C\left(\left[t_{0}, \infty\right),(0, \infty)\right), \quad r_{2}(t) \in C\left(\left[t_{0}, \infty\right),(0, \infty)\right), \quad m(t) \in C\left(\left[t_{0}, \infty\right),(0, \infty)\right)$,

$$
\int_{\mathrm{t}_{0}}^{\infty} \frac{1}{\mathrm{r}_{2}(\mathrm{t})} \mathrm{dt}=\infty, \quad \int_{\mathrm{t}_{0}}^{\infty} \frac{1}{\mathrm{r}_{1}(\mathrm{t})} \exp \left(-\int_{\mathrm{t}_{0}}^{\mathrm{t}} \frac{\mathrm{m}(\mathrm{s})}{\mathrm{r}_{1}(\mathrm{~s})} \mathrm{ds}\right) \mathrm{dt}=\infty
$$

*Corresponding author

Email address: wei_meihua@163.com (M. H. Wei)

doi: $10.22436 /$ jmcs.019.01.03 
$\left(C_{2}\right) p(t, \mu) \in C\left(\left[t_{0}, \infty\right) \times[a, b],(0, \infty)\right), \quad 0 \leqslant p(t) \equiv \int_{a}^{b} p(t, \mu) d \mu \leqslant p<1 ;$

$\left(C_{3}\right) \sigma(t, \mu) \in C\left(\left[t_{0}, \infty\right) \times[a, b],(0, \infty)\right)$ and $\tau(t, \zeta) \in C\left(\left[t_{0}, \infty\right) \times[c, d],(0, \infty)\right)$ are not the decreasing functions with respect to $\mu$ and $\zeta$, respectively, and satisfy $\sigma(t, \mu) \leqslant t, \tau(t, \zeta) \leqslant t, \lim _{t \rightarrow \infty} \inf _{\mu \in[a, b]} \sigma(t, \mu)=\infty$ and $\lim _{t \rightarrow \infty} \inf _{\zeta \in[c, d]} \tau(t, \zeta)=\infty ;$

$\left(C_{4}\right) q(t, \zeta) \in C\left(\left[t_{0}, \infty\right) \times[c, d],(0, \infty)\right) ;$

$\left(C_{5}\right) f(v) \in C((0, \infty),(0, \infty)), \quad \frac{f(v)}{v} \geqslant \alpha>0, \quad v \neq 0$.

Define the function by

$$
z(t)=x(t)+\int_{a}^{b} p(t, \mu) x(\sigma(t, \mu)) d \mu .
$$

By a solution of (1.1) this means a function $x(t) \in C\left(\left[T_{x}, \infty\right)\right)$ which has the property $x(t), r_{2}(t) z^{\prime}(t)$, $r_{1}(t)\left(r_{2}(t) z^{\prime}(t)\right)^{\prime} \in C^{1}\left[T_{x}, \infty\right)$ and satisfies (1.1) on $\left[T_{x}, \infty\right)$. Our attention is restricted to those solutions $x(t)$ of (1.1) which satisfy $\sup \left\{|x(t)|: t_{1} \leqslant t \leqslant \infty\right\}>0$ for all $t_{1} \geqslant t_{0}$. A solution of (1.1) is called oscillatory if it has arbitrarily large zeros on $\left[T_{x}, \infty\right)$ and otherwise it is called nonoscillatory.

Motivated by $[4,8,10,18,21,24]$, the study focuses on the oscillation behavior of neutral distributed delay differential equations with damping. The oscillation of functional differential equations have received a great deal of interest in recent years. But we notice that most of the investigations are concerned with oscillation of first or second order differential equations, while relatively less attention has been paid to oscillation of third order differential equations, see [1-3, 5-7, 9, 11-16, 18, 20, 22, 24], especially the distributed delay equations with damping. On the basis of the studies [18] and [24], by using a generalized Riccati transformation and choosing appropriate functions, the aim of this paper is to establish some new sufficient conditions which insure that any solution of this equation oscillates or converges to zero. In fact, by choosing appropriate functions, we shall present several easily verifiable oscillation criteria. The methods and arguments used in the present paper are different from those used in $[4,8,10,21]$, and the results correct, extend and improve a number of existing results, especially the results in [18] and [24].

The paper is organized as follows. We first need to state and prove some lemmas in Section 2, which will be used in the proof of our main results. We will establish some new criteria of oscillatory behavior for (1.1) by a generalized Riccati transformation technique in Section 3, and then present some applications for our results in Section 4.

\section{Some preliminary lemmas}

Lemma 2.1. Let $x(t)$ be a positive solution of (1.1). Then $z(t)$ has only one of the following two properties:

(I) $z(\mathrm{t})>0, z^{\prime}(\mathrm{t})>0,\left(\mathrm{r}_{2}(\mathrm{t}) z^{\prime}(\mathrm{t})\right)^{\prime}>0$;

(II) $z(\mathrm{t})>0, z^{\prime}(\mathrm{t})<0,\left(\mathrm{r}_{2}(\mathrm{t}) z^{\prime}(\mathrm{t})\right)^{\prime}>0$,

where $\mathrm{t} \geqslant \mathrm{t}_{1}$ for sufficiently large $\mathrm{t}_{1}$.

Proof. Let $x(t)$ be a positive solution of $(1.1)$ on $\left[t_{0}, \infty\right)$. Then we have $z(t)>x(t)>0$ from $\left(C_{2}\right)$ for $t \geqslant t_{1}$. Based on (1.2), it follows from $\left(C_{3}\right)$ and $\left(C_{4}\right)$ that

$$
\begin{aligned}
\left(r_{1}(t)\left(r_{2}(t) z^{\prime}(t)\right)^{\prime}\right)^{\prime}+m(t)\left(r_{2}(t) z^{\prime}(t)\right)^{\prime} & =-\int_{c}^{d} q(t, \zeta) f(x(\tau(t, \zeta))) d \zeta \\
& \leqslant-\alpha \int_{c}^{d} q(t, \zeta) x(\tau(t, \zeta)) d \zeta \\
& <0 .
\end{aligned}
$$


Then we have

$$
\frac{d}{d t}\left[\exp \left(\int_{t_{1}}^{t} \frac{m(s)}{r_{1}(s)} d s\right) r_{1}(t)\left(r_{2}(t) z^{\prime}(t)\right)^{\prime}\right]<0
$$

Thus, $\exp \left(\int_{t_{1}}^{t} \frac{m(s)}{r_{1}(s)} d s\right) r_{1}(t)\left(r_{2}(t) z^{\prime}(t)\right)^{\prime}$ is a decreasing function and eventually of one sign, and then from $\left(\mathrm{C}_{1}\right)$ we know that

$$
\left(\mathrm{r}_{2}(\mathrm{t}) z^{\prime}(\mathrm{t})\right)^{\prime}<0 \text { or }\left(\mathrm{r}_{2}(\mathrm{t}) z^{\prime}(\mathrm{t})\right)^{\prime}>0,
$$

for $t \geqslant t_{2} \geqslant t_{1}$. We assert that $\left(r_{2}(t) z^{\prime}(t)\right)^{\prime}>0$. Suppose $\left(r_{2}(t) z^{\prime}(t)\right)^{\prime}<0$, then there exists a constant $M>0$ such that

$$
\exp \left(\int_{t_{1}}^{t} \frac{m(s)}{r_{1}(s)} d s\right) r_{1}(t)\left(r_{2}(t) z^{\prime}(t)\right)^{\prime} \leqslant-M .
$$

Integrate the above inequality on $\left[t_{2}, t\right]$ to get

$$
r_{2}(t) z^{\prime}(t) \leqslant r_{2}\left(t_{2}\right) z^{\prime}\left(t_{2}\right)-M\left(\int_{t_{2}}^{t} \frac{1}{r_{1}(s)} \exp \left(-\int_{t_{1}}^{s} \frac{m(\eta)}{r_{1}(\eta)} d \eta\right) d s \rightarrow-\infty(t \rightarrow \infty),\right.
$$

by the condition $\left(C_{1}\right)$. Thus, there are an integer $t_{3} \geqslant t_{2}$ and $C>0$ such that $r_{2}(t) z^{\prime}(t) \leqslant-C$ for $t \geqslant t_{3}$. Integrating on $\left[t_{3}, t\right]$ to get

$$
z(t) \leqslant z\left(t_{3}\right)-C \int_{t_{3}}^{t} \frac{1}{r_{2}(s)} d s \rightarrow-\infty(t \rightarrow \infty),
$$

from the condition $\left(C_{1}\right)$, and then $z(t)<0$ which contradicts $z(t)>0$. Thus we have $\left(r_{2}(t) z^{\prime}(t)\right)^{\prime}>0$. Therefore, $z(t)$ has only one of the two properties (I) and (II). This completes the proof.

Lemma 2.2. Let $x(\mathrm{t})$ be a positive solution of (1.1), and $z(\mathrm{t})$ has the property (I). Then

$$
z^{\prime}(\tau(t)) \geqslant \frac{r_{1}(t)\left(r_{2}(t) z^{\prime}(t)\right)^{\prime} R(\tau(t))}{r_{2}(\tau(t))}
$$

where $\tau(t)=\tau(t, c), R(t)=\int_{t_{0}}^{t} \frac{1}{r_{1}(s)} d s, t \geqslant t_{0}$.

Proof. Let $x(t)$ be a positive solution of (1.1). Since $z(t)$ has the property $(I)$, we know

$$
\left(r_{1}(t)\left(r_{2}(t) z^{\prime}(t)\right)^{\prime}\right)^{\prime}<0,
$$

from (2.1). Then we have

$$
\begin{aligned}
r_{2}(t) z^{\prime}(t) & =r_{2}\left(t_{0}\right) z^{\prime}\left(t_{0}\right)+\int_{t_{0}}^{t}\left(r_{2}(s) z^{\prime}(s)\right)^{\prime} d s \\
& \geqslant \int_{t_{0}}^{t} \frac{r_{1}(s)\left(r_{2}(s) z^{\prime}(s)\right)^{\prime}}{r_{1}(s)} d s \\
& \geqslant r_{1}(t)\left(r_{2}(t) z^{\prime}(t)\right)^{\prime} R(t) .
\end{aligned}
$$

Thus

$$
\begin{aligned}
r_{2}(\tau(t)) z^{\prime}(\tau(t)) & \geqslant r_{1}(\tau(t))\left(r_{2}(\tau(t)) z^{\prime}(\tau(t))\right)^{\prime} R(\tau(t)) \\
& \geqslant r_{1}(t)\left(r_{2}(t) z^{\prime}(t)\right) R(\tau(t)) .
\end{aligned}
$$

Hence, we obtain

$$
z^{\prime}(\tau(t)) \geqslant \frac{r_{1}(t)\left(r_{2}(t) z^{\prime}(t)\right)^{\prime} R(\tau(t))}{r_{2}(\tau(t))}
$$

which completes the proof. 
Lemma 2.3. Let $x(t)$ be a positive solution of (1.1), and $z(t)$ has the property (II). If

$$
\int_{\mathrm{t}_{0}}^{\infty} \frac{1}{r_{2}(v)} \int_{v}^{\infty} \frac{1}{r_{1}(u)} \int_{u}^{\infty} \int_{c}^{d} q(s, \zeta) d \zeta d s d u d v=\infty
$$

then $\lim _{t \rightarrow \infty} x(t)=0$.

Proof. Let $x(t)$ be a positive solution of (1.1). According to the property (II), we have $z(t)>0, z^{\prime}(t)<0$, then $\lim _{t \rightarrow \infty} z(t)=l \geqslant 0$. We assert that $l=0$. Suppose that $l>0$, then we get $l+\varepsilon>z(t)>l$ for $\varepsilon>0$ and $t \geqslant t_{1} \geqslant t_{0}$. By choosing $\varepsilon<\frac{l(1-p)}{p}$, from $\left(C_{2}\right)$ and property (II) we have

$$
\begin{aligned}
x(t) & =z(t)-\int_{a}^{b} p(t, \mu) x(\sigma(t, \mu)) d \mu \\
& \geqslant z(t)-z(\sigma(t, a)) \int_{a}^{b} p(t, \mu) d \mu \\
& \geqslant l-p(t) z(\sigma(t, a)) \\
& \geqslant l-p(l+\varepsilon) \\
& >k z(t),
\end{aligned}
$$

where $k=\frac{l(1-p)-p \varepsilon}{l+\varepsilon}>0$. Then from $\left(C_{5}\right)$, we get

$$
\begin{aligned}
\left(r_{1}(t)\left(r_{2}(t) z^{\prime}(t)\right)^{\prime}\right)^{\prime}+m(t)\left(r_{2}(t) z^{\prime}(t)\right)^{\prime} & \leqslant-\alpha \int_{c}^{d} q(t, \zeta) x(\tau(t, \zeta)) d \zeta \\
& \leqslant-k \alpha z(\tau(t, d)) \int_{c}^{d} q(t, \zeta) d \zeta \\
& =-q_{1}(t) z\left(\tau_{1}(t)\right),
\end{aligned}
$$

where $q_{1}(t)=k \alpha \int_{c}^{d} q(t, \zeta) d \zeta, \tau_{1}(t)=\tau(t, d)$. Thus

$$
\left(\exp \left(\int_{t_{1}}^{t} \frac{m(s)}{r_{1}(s)} d s\right) r_{1}(t)\left(r_{2}(t) z^{\prime}(t)\right)^{\prime}\right)^{\prime} \leqslant-\exp \left(\int_{t_{1}}^{t} \frac{m(s)}{r_{1}(s)} d s\right) q_{1}(t) z\left(\tau_{1}(t)\right) .
$$

Integrating the above inequality from $t$ to $\infty$, we obtain

$$
r_{1}(t)\left(r_{2}(t) z^{\prime}(t)\right)^{\prime} \geqslant \exp \left(-\int_{t_{1}}^{t} \frac{m(s)}{r_{1}(s)} d s\right) \int_{t}^{\infty} \exp \left(\int_{t_{1}}^{\sigma} \frac{m(s)}{r_{1}(s)} d s\right) q_{1}(\sigma) z\left(\tau_{1}(\sigma)\right) d \sigma .
$$

From $z\left(\tau_{1}(t)\right)>l$ and $\frac{d}{d t} \exp \left(\int_{t_{1}}^{t} \frac{m(s)}{r_{1}(s)} d s\right)>0$, we have

$$
\left(r_{2}(t) z^{\prime}(t)\right)^{\prime}>\frac{l}{r_{1}(t)} \int_{t}^{\infty} q_{1}(s) d s .
$$

Integrate the above inequality on $[t, \infty)$ to get

$$
-r_{2}(t) z^{\prime}(t)>l \int_{t}^{\infty} \frac{1}{r_{1}(u)} \int_{u}^{\infty} q_{1}(s) d s d u
$$

Further integrating on $\left[t_{1}, \infty\right)$ leads to

$$
\int_{t_{1}}^{\infty} \frac{1}{r_{2}(v)} \int_{v}^{\infty} \frac{1}{r_{1}(u)} \int_{u}^{\infty} \int_{c}^{d} q(s, \zeta) d \zeta d s d u d v<\frac{z\left(t_{1}\right)}{k \alpha l}
$$

which contradicts (2.2), and then we have $l=0$. According to $z(t)>x(t)>0$, we obtain $\lim _{t \rightarrow \infty} x(t)=0$. 


\section{Main results}

In this section, we obtain new oscillatory criteria for (1.1) by using the generalized Riccati transformation. Let

$$
\mathrm{D}=\left\{(\mathrm{t}, \mathrm{s}): \mathrm{t}_{0} \leqslant \mathrm{~s} \leqslant \mathrm{t}<\infty\right\}, \quad \mathrm{D}_{0}=\left\{(\mathrm{t}, \mathrm{s}): \mathrm{t}_{0} \leqslant \mathrm{~s}<\mathrm{t}<\infty\right\} .
$$

A function $H \in C^{1}(D, R)$ is said to belong to $X$ class $(H \in X)$ if it satisfies

(i) $H(t, t)=0, t \geqslant t_{0}, \quad H(t, s)>0,(t, s) \in D_{0}$;

(ii) $\frac{\partial H(t, s)}{\partial s} \leqslant 0,(t, s) \in D$, and there exists $h(t, s) \in C\left(D_{0}, R\right)$ such that

$$
\frac{\partial H(t, s)}{\partial s}=-h(t, s) \sqrt{H(t, s)}
$$

Then we present the following main results of this article.

Theorem 3.1. Assume that (2.2) holds. If there exists $\rho(t) \in C^{1}\left(\left[t_{0}, \infty\right),(0, \infty)\right)$ such that

$$
\limsup _{t \rightarrow \infty} \int_{t_{0}}^{t}\left(C(s)-\frac{B^{2}(s)}{4 A(s)}\right) d s=\infty
$$

where

$$
A(t)=\frac{\tau^{\prime}(t) R(\tau(t))}{\rho(t) r_{2}(\tau(t))}, \quad B(t)=\frac{\rho^{\prime}(t)}{\rho(t)}-\frac{m(t)}{r_{1}(t)}, \quad C(t)=\alpha(1-p) \rho(t) q(t),
$$

and $\mathrm{q}(\mathrm{t})=\int_{\mathcal{c}}^{\mathrm{d}} \mathrm{q}(\mathrm{t}, \zeta) \mathrm{d} \zeta$, then every solution $\mathrm{x}(\mathrm{t})$ of (1.1) either oscillates or converges to zero.

Proof. Let $x(t)$ be a nonoscillatory solution of (1.1). Without loss of generality, we may assert $x(t)>0$ on $\left[t_{1}, \infty\right)$, and then $x(\sigma(t, \mu))>0,(t, \mu) \in\left[t_{1}, \infty\right) \times[a, b], x(\tau(t, \zeta))>0,(t, \zeta) \in\left[t_{1}, \infty\right) \times[c, d]$ for sufficiently large $t_{1}$. By Lemma 2.1, we know that $z(t)$ has the property (I) or the property (II).

When $z(t)$ has property $(I)$, it follows from $\left(C_{2}\right)$ and $\left(C_{3}\right)$, we have

$$
\begin{aligned}
x(t) & =z(t)-\int_{a}^{b} p(t, \mu) x(\sigma(t, \mu)) d \mu \\
& \geqslant z(t)-\int_{a}^{b} p(t, \mu) z(\sigma(t, \mu)) d \mu \\
& \geqslant(1-p(t)) z(t) \\
& \geqslant(1-p) z(t) .
\end{aligned}
$$

Then

$$
\begin{aligned}
\left(r_{1}(t)\left(r_{2}(t) z^{\prime}(t)\right)^{\prime}\right)^{\prime}+m(t)\left(r_{2}(t) z^{\prime}(t)\right)^{\prime} & \leqslant-\alpha(1-p) \int_{c}^{d} q(t, \zeta) z(\tau(t, \zeta)) d \zeta \\
& \leqslant-\alpha(1-p) z(\tau(t)) q(t)
\end{aligned}
$$

Let

$$
w(t)=\rho(t) \frac{r_{1}(t)\left(r_{2}(t) z^{\prime}(t)\right)^{\prime}}{z(\tau(t))}, t \geqslant t_{1}
$$


Then from Lemma 2.2, we obtain

$$
\begin{aligned}
w^{\prime}(t)= & \rho^{\prime}(t) \frac{r_{1}(t)\left(r_{2}(t) z^{\prime}(t)\right)^{\prime}}{z^{\prime}(\tau(t))}+\rho(t) \frac{\left(r_{1}(t)\left(r_{2}(t) z^{\prime}(t)\right)^{\prime}\right)^{\prime}}{z(\tau(t))}-\rho(t) \frac{z^{\prime}(\tau(t)) \tau^{\prime}(t) r_{1}(t)\left(r_{2}(t) z^{\prime}(t)\right)^{\prime}}{z^{2}(\tau(t))} \\
\leqslant & \frac{\rho^{\prime}(t)}{\rho(t)} \mathcal{w}(t)-\rho(t)\left[\frac{m(t)\left(r_{2}(t) z^{\prime}(t)\right)^{\prime}}{z(\tau(t))}+\frac{\alpha(1-p) z(\tau(t)) q(t)}{z(\tau(t))}\right] \\
& -\rho(t) \frac{\tau^{\prime}(t) R(\tau(t))\left(r_{1}(t)\left(r_{2}(t) z^{\prime}(t)\right)^{\prime}\right)^{2}}{r_{2}(\tau(t)) z^{2}(\tau(t))} \\
\leqslant & -C(t)+B(t) w(t)-A(t) w^{2}(t) \\
\leqslant & -C(t)+\frac{B^{2}(t)}{4 A(t)}
\end{aligned}
$$

Integrate the above inequality from $t_{2}$ to $t$, then we have

$$
\int_{t_{1}}^{t}\left(C(s)-\frac{B^{2}(s)}{4 A(s)}\right) d s \leqslant w\left(t_{2}\right)
$$

from $w(t)>0$, which contradicts (3.1), and then the solution $x(t)$ of $(1.1)$ is oscillatory.

When $z(t)$ has property (II), from (2.2) we know $\lim _{t \rightarrow \infty} x(t)=0$ by Lemma 2.3. The proof is complete.

Remark 3.2. The proof of Theorem 3.1 is based on $B u-A u^{2} \leqslant \frac{B^{2}}{4 A}$ for $A>0, u \in R$.

Theorem 3.3. Assume that (2.2) holds. If there exist $\mathrm{H} \in \mathrm{X}$ and $\rho(\mathrm{t}) \in \mathrm{C}^{1}\left(\left[\mathrm{t}_{0}, \infty\right),(0, \infty)\right)$ such that

$$
\limsup _{t \rightarrow \infty} \frac{1}{H\left(t, t_{0}\right)} \int_{t_{0}}^{t}\left(H(t, s) C(s)-\frac{h_{1}^{2}(t, s)}{4 A(s)}\right) d s=\infty,
$$

where

$$
h_{1}(t, s)=h(t, s)-B(s) \sqrt{H(t, s)},
$$

and $\mathrm{A}(\mathrm{t}), \mathrm{B}(\mathrm{t}), \mathrm{C}(\mathrm{t})$ are defined in (3.2), then every solution $\mathrm{x}(\mathrm{t})$ of (1.1) either oscillates or converges to zero.

Proof. Let $x(t)$ be a nonoscillatory solution of (1.1). Without loss of generality, we may assert $x(t)>0, t \geqslant$ $t_{1} \geqslant t_{0}, x(\sigma(t, \mu))>0,(t, \mu) \in\left[t_{1}, \infty\right) \times[a, b], x(\tau(t, \zeta))>0,(t, \zeta) \in\left[t_{1}, \infty\right) \times[c, d]$. By Lemma 2.1, we know that $z(t)$ has property (I) or property (II).

When $z(t)$ has the property (I), we proceed as in the proof of Theorem 3.1 and have

$$
w^{\prime}(\mathrm{t}) \leqslant-\mathrm{C}(\mathrm{t})+\mathrm{B}(\mathrm{t}) w(\mathrm{t})-\mathrm{A}(\mathrm{t}) w^{2}(\mathrm{t})
$$

Multiplying the above inequality by $\mathrm{H}(\mathrm{t}, \mathrm{s})$ and integrating the inequality from $\mathrm{t}_{1}$ to $t$, we obtain

$$
\begin{aligned}
\int_{t_{1}}^{t} H(t, s) C(s) d s \leqslant & w\left(t_{1}\right) H\left(t, t_{1}\right)-\int_{t_{1}}^{t}\left(h_{1}(t, s) \sqrt{H(t, s)} w(s)+H(t, s) A(s) w^{2}(s)\right) d s \\
= & w\left(t_{1}\right) H\left(t, t_{1}\right)-\int_{t_{1}}^{t}\left(\sqrt{H(t, s) A(s)} w(s)+\frac{h_{1}(t, s)}{2 \sqrt{A(s)}}\right)^{2} d s \\
& +\int_{t_{1}}^{t} \frac{h_{1}^{2}(t, s)}{4 A(s)} d s
\end{aligned}
$$


where $h_{1}(t, s)$ is defined as (3.4). Then

$$
\frac{1}{\mathrm{H}\left(\mathrm{t}, \mathrm{t}_{1}\right)} \int_{\mathrm{t}_{1}}^{\mathrm{t}}\left(\mathrm{H}(\mathrm{t}, \mathrm{s}) \mathrm{C}(\mathrm{s})-\frac{\mathrm{h}_{1}^{2}(\mathrm{t}, \mathrm{s})}{4 \mathrm{~A}(\mathrm{~s})}\right) \mathrm{ds} \leqslant w\left(\mathrm{t}_{1}\right),
$$

which contradicts (3.3). Thus the solution $x(t)$ of (1.1) is oscillatory.

When $z(t)$ has property (II), from (2.2) we know $\lim _{t \rightarrow \infty} x(t)=0$ by Lemma 2.3. The proof is complete.

Theorem 3.4. Assume that (2.2) holds. If there exist $\mathrm{H} \in \mathrm{X}, \rho(\mathrm{t}) \in \mathrm{C}^{1}\left(\left[\mathrm{t}_{0}, \infty\right),(0, \infty)\right)$ and $\varphi \in \mathrm{C}\left(\left[\mathrm{t}_{0}, \infty\right), \mathrm{R}\right)$ such that

$$
\begin{gathered}
0<\inf _{s \geqslant T}\left[\liminf _{t \rightarrow \infty} \frac{H(t, s)}{H(t, T)}\right] \leqslant \infty, \\
\limsup _{t \rightarrow \infty} \frac{1}{H(t, T)} \int_{T}^{t} \frac{h_{1}^{2}(t, s)}{A(s)} d s<\infty, \\
\int_{t_{0}}^{\infty} A(t) \varphi_{+}^{2}(t) d t=\infty,
\end{gathered}
$$

and

$$
\varphi(T) \leqslant \limsup _{t \rightarrow \infty} \frac{1}{H(t, T)} \int_{T}^{t}\left(H(t, s) C(s)-\frac{h_{1}^{2}(t, s)}{4 A(s)}\right) d s,
$$

for $\mathrm{t} \geqslant \mathrm{T} \geqslant \mathrm{t}_{0}$, where

$$
\varphi_{+}(\mathrm{t})=\max \{\varphi(\mathrm{t}), 0\},
$$

$\mathrm{A}(\mathrm{t}), \mathrm{B}(\mathrm{t}), \mathrm{C}(\mathrm{t})$ and $\mathrm{h}_{1}(\mathrm{t}, \mathrm{s})$ are defined in (3.2) and (3.4), respectively, then every solution $\mathrm{x}(\mathrm{t})$ of (1.1) either oscillates or converges to zero.

Proof. Let $x(t)$ be a nonoscillatory solution of (1.1). Proceeding as in the proof of Theorem 3.3, when $z(t)$ has property (I), from (3.5) we have

$$
\begin{aligned}
\limsup _{t \rightarrow \infty} \frac{1}{H(t, T)} \int_{T}^{t}\left(H(t, s) C(s)-\frac{h_{1}^{2}(t, s)}{4 A(s)}\right) d s \\
\quad \leqslant w(T)-\liminf _{t \rightarrow \infty} \frac{1}{H(t, T)} \int_{T}^{t}\left(\sqrt{H(t, s) A(s)} w(s)+\frac{h_{1}(t, s)}{2 \sqrt{A(s)}}\right)^{2} d s .
\end{aligned}
$$

It follows from (3.7) that

$$
\varphi(T) \leqslant w(T)
$$

and then

$$
\liminf _{t \rightarrow \infty} \frac{1}{H(t, T)} \int_{T}^{t}\left(\sqrt{H(t, s) A(s)} w(s)+\frac{h_{1}(t, s)}{2 \sqrt{A(s)}}\right)^{2} d s \leqslant w(T)-\varphi(T)<\infty
$$

Thus we know

$$
\liminf _{t \rightarrow \infty} \frac{1}{H(t, T)} \int_{T}^{t}\left(H(t, s) A(s) w^{2}(s)+\sqrt{H(t, s)} h_{1}(t, s) w(s)\right) d s<\infty .
$$


Here we assert

$$
\int_{t_{1}}^{\infty} A(s) w^{2}(s) d s<\infty
$$

Suppose $\int_{t_{1}}^{\infty} A(s) w^{2}(s) d s=\infty$. From (3.6), we have

$$
\inf _{s \geqslant T}\left[\liminf _{t \rightarrow \infty} \frac{H(t, s)}{H(t, T)}\right]>\mu
$$

for $\mu>0$, then $\frac{H\left(t, t_{2}\right)}{H(t, T)}>\mu$ for $t \geqslant t_{2} \geqslant t_{1}$. There exists $M_{1}>0$ such that

$$
\int_{t_{1}}^{t} A(s) w^{2}(s) d s \geqslant \frac{M_{1}}{\mu}
$$

Thus for $t \geqslant t_{2}$,

$$
\begin{aligned}
\frac{1}{\mathrm{H}(\mathrm{t}, \mathrm{T})} \int_{\mathrm{t}_{2}}^{\mathrm{t}} \mathrm{H}(\mathrm{t}, \mathrm{s}) \mathrm{A}(\mathrm{s}) w^{2}(\mathrm{~s}) \mathrm{d} s & =\frac{1}{\mathrm{H}(\mathrm{t}, \mathrm{T})} \int_{\mathrm{t}_{2}}^{\mathrm{t}}-\frac{\partial \mathrm{H}(\mathrm{t}, \mathrm{s})}{\partial \mathrm{s}} \int_{\mathrm{t}_{2}}^{\mathrm{s}} \mathrm{A}(\eta) w^{2}(\eta) \mathrm{d} \eta \mathrm{d} s \\
& \geqslant \frac{1}{\mathrm{H}(\mathrm{t}, \mathrm{T})} \frac{\mathrm{M}_{1}}{\mu} \int_{\mathrm{t}_{2}}^{\mathrm{t}}-\frac{\partial \mathrm{H}(\mathrm{t}, \mathrm{s})}{\partial \mathrm{s}} \mathrm{ds} \\
& =\frac{\mathrm{M}_{1}}{\mu} \frac{\mathrm{H}\left(\mathrm{t}, \mathrm{t}_{2}\right)}{\mathrm{H}(\mathrm{t}, \mathrm{T})} \\
& \geqslant M_{1} .
\end{aligned}
$$

Hence, we have

$$
\liminf _{t \rightarrow \infty} \frac{1}{H(t, T)} \int_{T}^{t} H(t, s) A(s) w^{2}(s) d s=\infty
$$

The reminder of the proof is similar to that of similar theorems in $[17,19,23]$ and hence is omitted. Then we get that the solution $x(t)$ of $(1.1)$ is oscillatory.

When $z(t)$ has property (II), from (2.2) we find $\lim _{t \rightarrow \infty} x(t)=0$ by Lemma 2.3. The proof is complete.

\section{Examples}

In this section, we will give several examples to illustrate our main results.

Example 4.1. We consider the equation

$$
\begin{aligned}
& \left(\frac{1}{(t+1)^{2}}\left(x(t)+\int_{-1}^{0}\left(\frac{1}{2}+\frac{2}{3} e^{-2 t}+\mu\right) x\left(t+\frac{\mu}{2}\right) d \mu\right)^{\prime}\right)^{\prime \prime} \\
& \quad+\frac{1}{t}\left(\frac{1}{(t+1)^{2}}\left(x(t)+\int_{-1}^{0}\left(\frac{1}{2}+\frac{2}{3} e^{-2 t}+\mu\right) x\left(t+\frac{\mu}{2}\right) d \mu\right)^{\prime}\right)^{\prime \prime} \\
& \quad+\int_{-1}^{0} e^{t+\zeta}(3+\sin x(t+\zeta)) x(t+\zeta) d \zeta=0, \quad t>1
\end{aligned}
$$

Based on (4.1), we find that $r_{1}(t)=1, r_{2}(t)=\frac{1}{(t+1)^{2}}, p(t, \mu)=\frac{1}{2}+\frac{2}{3} e^{-2 t}+\mu, p=\frac{2}{3}, a=c=-1, b=$ $\mathrm{d}=0, \mathrm{t}_{0}=1, \sigma(\mathrm{t}, \mu)=\mathrm{t}+\frac{\mu}{2} \leqslant \mathrm{t}, \mu \in[-1,0], \mathrm{m}(\mathrm{t})=\frac{1}{\mathrm{t}}, \tau(\mathrm{t}, \zeta)=\mathrm{t}+\zeta \leqslant \mathrm{t}, \zeta \in[-1,0], \mathrm{q}(\mathrm{t}, \zeta)=$ 
$e^{t+\zeta}, f(v)=(3+\sin v) v, \alpha=2$. It is clear that the conditions $\left(C_{1}\right)-\left(C_{5}\right)$ are satisfied. By choosing $H(t, s)=(t-s)^{2}, \rho(t)=1$, from Theorem 3.3 we have $h(t, s)=2, q(t)=\left(1-e^{-1}\right) e^{t}, R(t)=t-1, \tau(t)=$ $t-1, A(t)=t^{2}(t-2), B(t)=-\frac{1}{t}, C(t)=\frac{2\left(1-e^{-1}\right)}{3} e^{t}, h_{1}(t, s)=1+\frac{t}{s}$, and

$$
\limsup _{t \rightarrow \infty} \frac{1}{H\left(t, t_{0}\right)} \int_{t_{0}}^{t}\left(H(t, s) C(s)-\frac{h_{1}^{2}(t, s)}{4 A(s)}\right) d s=\infty \text {. }
$$

Hence by Theorem 3.3, every solution $x(t)$ of (1.1) is oscillatory or $x(t) \rightarrow 0$ as $t \rightarrow \infty$.

Example 4.2. Consider the equation

$$
\begin{aligned}
& \left(e^{-t}\left(x(t)+\int_{1}^{2} \frac{\mu}{3 t} x\left(\frac{1}{2} t \mu\right) d \mu\right)^{\prime}\right)^{\prime \prime}+\frac{1}{t}\left(e^{-t}\left(x(t)+\int_{1}^{2} \frac{\mu}{3 t} x\left(\frac{1}{2} t \mu\right) d \mu\right)^{\prime}\right)^{\prime \prime} \\
& +\int_{0}^{1} t \zeta x(t-\zeta) d \zeta=0, \quad t>1
\end{aligned}
$$

Here from (4.2), we note that $r_{1}(t)=1, r_{2}(t)=e^{-t}, p(t, \mu)=\frac{\mu}{3 t}, p=\frac{1}{2}, a=1, b=2, c=0, d=1, t_{0}=$ $1, \sigma(t, \mu)=\frac{1}{2} \mathrm{t} \mu \leqslant \mathrm{t}, \mu \in[1,2], \mathrm{m}(\mathrm{t})=\frac{1}{\mathrm{t}}, \tau(\mathrm{t}, \zeta)=\mathrm{t}-\zeta \leqslant \mathrm{t}, \zeta \in[0,1], \mathrm{q}(\mathrm{t}, \zeta)=\mathrm{t} \zeta, \mathrm{f}(v)=v, \alpha=1$. Consequently the conditions $\left(C_{1}\right)-\left(C_{5}\right)$ are satisfied. Choose $H(t, s)=(t-s)^{2}, \rho(t)=e^{t}, \varphi(t)=t$, then from Theorem 3.4 we have $h(t, s)=2, q(t)=\frac{t}{2}, R(t)=t-1, \tau(t)=t, A(t)=t-1, B(t)=1-\frac{1}{t}, C(t)=$ $\frac{1}{4} t e^{t}, h_{1}(t, s)=1+\frac{t}{s}-t+s$, and

$$
\limsup _{t \rightarrow \infty} \frac{1}{H(t, T)} \int_{T}^{t}\left(H(t, s) C(s)-\frac{h_{1}^{2}(t, s)}{4 A(s)}\right) d s \geqslant T=\varphi(T)
$$

Clearly, it is easy to check that the other conditions of Theorem 3.4 are also satisfied. Thus, we can conclude from Theorem 3.4 that every solution $x(t)$ of $(1.1)$ is oscillatory or $x(t) \rightarrow 0$ as $t \rightarrow \infty$.

\section{Acknowledgment}

This work was supported by the National Natural Science Foundation of China (No. 11501496), the Natural Science Basic Research Plan in Shaanxi Province of China (No. 2014JQ2-1003), and the Doctor Start-up Research Fund of Yulin University (No. 13GK04).

\section{References}

[1] R. P. Agarwal, M. Bohner, T. X. Li, C. H. Zhang, Hille and Nehari type criteria for third-order delay dynamic equations, J. Difference Equ. Appl., 19 (2013), 1563-1579. 1

[2] R. P. Agarwal, M. Bohner, T. X. Li, C. H. Zhang, Oscillation of third-order nonlinear delay differential equations, Taiwanese J. Math., 17 (2013), 545-558.

[3] M. F. Aktas, A. Tiryaki, A. Zafer, Oscillation criteria for third order nonlinear functional differential equations, Appl. Math. Lett., 23 (2010), 756-762. 1

[4] M. Bohner, S. R. Grace, I. Sağer, E. Tunç, Oscillation of third-order nonlinear damped delay differential equations, Appl. Math. Comput., 278 (2016), 21-32. 1

[5] L. Erbe, T. S. Hassan, A. Peterson, Oscillation of third order nonlinear functional dynamic equations on time scales, Differ. Equ. Dyn. Syst., 18 (2010), 199-227. 1

[6] L. Erbe, T. S. Hassan, A. Peterson, Oscillation of third-order functional dynamic equations with mixed arguments on time scales, J. Appl. Math. Comput., 34 (2010), 353-371.

[7] L. Erbe, A. Peterson, S. H. Saker, Hille and Nehari type criteria for third order dynamic equations, J. Math. Anal. Appl., 329 (2007), 112-131. 1 
[8] S. R. Grace, Oscillation criteria for third-order nonlinear delay differential equations with damping, Opuscula Math., 35 (2015), 485-497. 1

[9] S. R. Grace, J. R. Graef, M. A. El-Beltagy, On the oscillation of third order neutral delay dynamic equations on time scales, Comput. Math. Appl., 63 (2012), 775-782. 1

[10] S. R. Grace, J. R. Graef, E. Tunç, On the oscillation of certain third order nonlinear dynamic equations with a nonlinear damping term, Math. Slovaca, 67 (2017), 501-508. 1

[11] Z. L. Han, T. X. Li, S. R. Sun, F. J. Cao, Oscillation criteria for third order nonlinear delay dynamic equations on time scales, Ann. Polon. Math., 99 (2010), 143-156. 1

[12] T. S. Hassan, Oscillation of third order nonlinear delay dynamic equations on time scales, Math. Comput. Modelling, 49 (2009), 1573-1586.

[13] T. X. Li, Z. L. Han, S. R. Sun, Y. Zhao, Oscillation results for third order nonlinear delay dynamic equations on time scales, Bull. Malays. Math. Sci. Soc. (2), 34 (2011), 639-648.

[14] T. X. Li, Y. V. Rogovchenko, On asymptotic behavior of solutions to higher-order sublinear Emden-Fowler delay differential equations, Appl. Math. Lett., 67 (2017), 53-59.

[15] S. Padhi, S. Pati, Theory of third-order differential equations, Springer, New Delhi, (2014).

[16] Y.-C. Qiu, A. Zada, H. Y. Qin, T. X. Li, Oscillation criteria for nonlinear third-order neutral dynamic equations with damping on time scales, J. Funct. Spaces, 2017 (2017), 8 pages. 1

[17] Y. V. Rogovchenko, Oscillation theorems for second-order equations with damping, Nonlinear Anal., 41 (2000), 10051028. 3

[18] S. H. Saker, Oscillation criteria of third-order nonlinear delay differential equations, Math. Slovaca, 56 (2006), 433-450. 1

[19] S. H. Saker, P. Y. H. Pang, R. P. Agarwal, Oscillation theorems for second order functional differential equations with damping, Dynam. Systems Appl., 12 (2003), 307-321. 3

[20] Y. B. Sun, Z. Han, Y. Sun, Y. Pan, Oscillation theorems for certain third order nonlinear delay dynamic equations on time scales, Electron. J. Qual. Theory Differ. Equ., 75 (2011), 1-14. 1

[21] A. Tiryaki, M. F. Aktas, Oscillation criteria of a certain class of third order nonlinear delay differential equations with damping, J. Math. Anal. Appl., 325 (2007), 54-68. 1

[22] A. Tiryaki, S. (Yaman) Pasinliogu, Oscillatory behaviour of a class of nonlinear differential equations of third order, Acta Math. Sci. Ser. B Engl. Ed., 21 (2001), 182-188. 1

[23] A. Tiryaki, A. Zafer, Oscillation criteria for second-order nonlinear differential equations with damping, Turkish J. Math., 24 (2000), 185-196. 3

[24] Q. X. Zhang, L. Gao, Y. H. Yu, Oscillation criteria for third-order neutral differential equations with continuously distributed delay, Appl. Math. Lett., 25 (2012), 1514-1519. 1 